\title{
NetFPGA - Rapid Prototyping of High Bandwidth Devices in Open Source
}

\author{
Noa Zilberman, Yury Audzevich, Georgina Kalogeridou, Neelakandan Manihatty Bojan, \\ Jingyun Zhang, Andrew W. Moore \\ University of Cambridge, Email: firstname.lastname@cl.cam.ac.uk
}

\begin{abstract}
The demand-led growth of datacenter networks has meant that many constituent technologies are beyond the budget of the wider community. In order to make and validate timely and relevant new contributions, the wider community requires accessible evaluation, experimentation and demonstration environments with specification comparable to the subsystems of the most massive datacenter networks. We demonstrate NetFPGA SUME, an open-source FPGA-based PCIe board for rapid prototyping of high bandwidth devices. NetFPGA SUME has I/O capabilities for $100 \mathrm{Gbps}$ operation as a networking device, computing unit, or for test and measurement.
\end{abstract}

\section{THE NETFPGA PROJECT}

The NetFPGA project (www.netfpga.org) provides software, hardware and community as a basic infrastructure to simplify design, simulation and testing, all around an open-source highspeed networking platform. Beyond the hardware and software, the NetFPGA project is backed by community resources that include online forums, tutorials, summer camp events and developer workshops, all supported by the NetFPGA project team. As all the (reference) projects developed under the NetFPGA project are open-source, by reusing building blocks across projects users compare design utilization and performance. Reference projects, included in all NetFPGA distributions, are a NIC, a switch and an IPv4 router.

\section{NETFPGA SUME}

The NetFPGA SUME [1] design aims to create a low-cost, PCIe host adapter card able to support $40 \mathrm{~Gb} / \mathrm{s}$ and $100 \mathrm{~Gb} / \mathrm{s}$ applications. At the core of the board is a Xilinx Virtex-7 690T FPGA device. There are five peripheral subsystems that complement the FPGA. A high-speed serial interfaces subsystem composed of 30 serial links running at up to $13.1 \mathrm{~Gb} / \mathrm{s}$. These connect four $10 \mathrm{~Gb} / \mathrm{s}$ SFP+ Ethernet interfaces, two expansion connectors and a PCIe edge connector directly to the FPGA. The second subsystem, the latest generation 3.0 of PCle is used to interface between the card and the host device, allowing both register access and packet transfer between the platform and the motherboard. The memory subsystem combines both SRAM and DRAM devices. SRAM memory is devised from three 36-bit QDRII+ devices, running at $500 \mathrm{MHz}$. The DRAM memory is composed of two 64bit, $933 \mathrm{MHz}(1866 \mathrm{MT} / \mathrm{s})$ DDR3 memory modules. Storage subsystems of the design permit both a MicroSD card and external disks through two SATA interfaces. Finally, the FPGA configuration subsystem is concerned with use of the FLASH devices. Additional NetFPGA SUME features support debug, extension and synchronization of the board. The board is implemented as a dual-slot, full-size PCIe adapter, that can

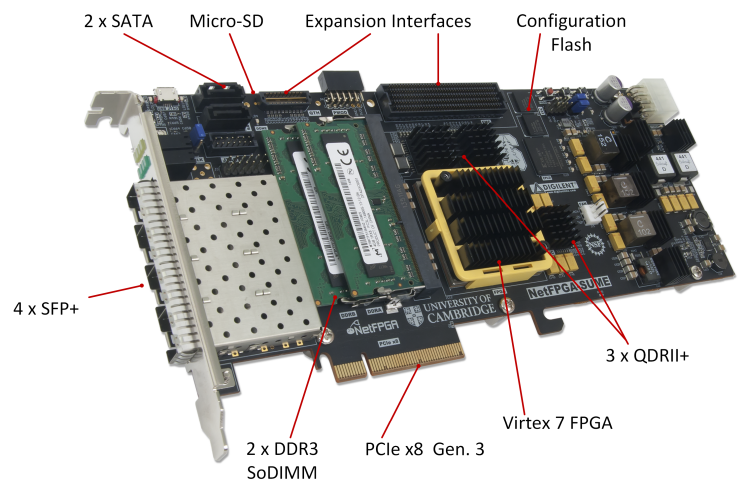

Fig. 1. NetFPGA SUME Board

operate as a standalone unit outside of a PCIe host. It is manufactured by Digilent Inc.(http://www.digilentinc.com).

\section{DEMONSTRATION}

We present the NetFPGA SUME platform, the NetFPGA development environment and a reference application exercising all of NetFPGA SUME interfaces. We show the development flow over the platform: code development, simulation environment and test harness, and demonstrate the rapid prototyping of a project. The NetFPGA project also provides access to an open-source code repository, online documentation and user forums, and debug tools. We provide a glimpse into the use of these tools under the demonstrated application, showing the performance of the hardware interfaces.

\section{Acknowledgements}

This work was jointly supported by EPSRC INTERNET Project EP/H040536/1, National Science Foundation under Grant No. CNS-0855268, and Defense Advanced Research Projects Agency (DARPA) and Air Force Research Laboratory (AFRL), under contract FA8750-11-C-0249. The views, opinions, and/or findings contained in this report are those of the authors and should not be interpreted as representing the official views or policies, either expressed or implied, of the National Science Foundation, Defense Advanced Research Projects Agency or the Department of Defense.

The Xilinx XUP program has been a long-standing supporter of NetFPGA and the NetFPGA SUME project is only possible with their generous support. We thank the people at Digilent Inc. We thank Micron and Cypress Semiconductor for their generous part donations.

\section{REFERENCES}

[1] N. Zilberman, Y. Audzevich, G. A. Covington, and A. W. Moore, "NetFPGA SUME: Toward 100 Gbps as Research Commodity," IEEE Micro, no. 5, pp. 32-41, 2014. 